ISSN 1808-3765

\title{
ESTUDO COMPARATIVO DE MÉTODOS PARA A DETERMINAÇÃO DA CURVA DE RETENÇÃO DE ÁGUA NO SOLO
}

\author{
Patricia dos Santos Nascimento ${ }^{1}$; Luís Henrique Bassoi ${ }^{2}$; Vital Pedro da Silva Paz $^{3}$; \\ Carlos Manoel Pedro $\mathrm{Vaz}^{4}$; João de Mendonça Naime ${ }^{4}$; Juliana Maria Manieri ${ }^{4}$ \\ ${ }^{I}$ Universidade Federal do Recôncavo da Bahia, Cruzdas Almas, Bahia.patyysn@yahoo.com.br \\ ${ }^{2}$ Embrapa Semi-Árido, Petrolina, Pernambuco \\ ${ }^{3}$ Universidade Federal do Recôncavo da Bahia, Cruz das Almas, Bahia \\ ${ }^{4}$ Embrapa Instrumentação Agropecuária, São Carlos, São Paulo.
}

\section{RESUMO}

A curva de retenção de água no solo pode ser estimada por diversos métodos, e alguns deles demandam maior tempo para a sua determinação. Assim, o objetivo desse trabalho foi a comparação da curva de retenção de água no solo, determinada pelos métodos de Arya \& Paris, câmara de Richards e centrífuga, em um Neossolo Quartzarênico em Petrolina - PE. Nas camadas de 0,00-0,20, 0,20-0,40 e 0,40-0,60 m de profundidade, foram coletadas amostras deformadas em 3 pontos de uma área cultivada com videiras irrigadas, as quais foram homogeneizadas por camada, formando assim uma amostra composta para cada camada; em seguida, tais amostras foram subdividas em três subamostras e cada uma foi encaminhada para a determinação da curva de retenção de água no solo pelos métodos testados. Os resultados obtidos pelo método de Arya \& Paris não apresentaram correspondência com os obtidos pelos métodos da centrífuga e da câmara de Richards. No entanto, o desenvolvimento de calibrações específicas do método de Arya \& Paris para os solos irrigados do Submédio São Francisco é recomendada, tanto pela possibilidade de uso da curva de retenção de água no solo para o manejo de irrigação, como pelo potencial do método quanto à determinação rápida.

UNITERMOS: retenção de água do solo, analisador granulométrico, método.

\section{NASCIMENTO, P. dos S.; BASSOI, L. H.; PAZ, V. P. da S.; VAZ, C. M. P.; NAIME, J. de M.; MANIERI, J. M. COMPARATIVE STUDY OF DETERMINING METHODS OF SOIL WATER RETENTION CURVE}

\section{ABSTRACT}

Soil water retention curve can be estimated by different methods, and some of them are time consuming. Hence, this research aimed to determine and compare the soil water retention curve, obtained by the methods proposed by Arya \& Paris, Richards (pressure membrane apparatus) and centrifuge, of a Typic Quartzipisamment from Petrolina, State of Pernambuco, Brazil. To determine the soil water retention curve in the layers of $0.00-0.20 ; 0.20-0.40$ and 0.40-0.60 m depths, disturbed soil samples were collected in three points of an irrigated vineyard area. The disturbed soil samples were homogenized by layer, thus forming a composed sample for each soil layer. These samples were subdivided into three sub samples, and each one was used to determination of soil water retention curve by the methods tested. 
The results from method proposed by Arya \& Paris did not present similarity with those obtained by Richards'chamber and centrifuge methods. Nevertheless, the development of specific calibration to irrigated soils from Lower-middle São Francisco region is recommended due to the application of soil water retention curve to irrigation scheduling as well as the quickness of the method.

KEYWORDS: soil water retention, granulometry analyzer, method.

\section{INTRODUÇÃO}

A curva de retenção da água no solo expressa a relação entre o potencial mátrico e a umidade do solo. A retenção de água na matriz do solo é governada por forças capilares e forças de adsorção, as quais são denominadas de forças mátricas, dando origem ao termo potencial mátrico da água no solo (Vieira, 2006). O conhecimento do potencial de água no solo é decisivo no estabelecimento das necessidades de irrigação para a cultura, permitindo definir a dedução máxima da água no solo no intervalo de água disponível as plantas (Marciano, 1998).

Alguns fatores têm relevante importância na retenção de água por um solo, porém, a textura merece uma atenção especial, sendo o seu conhecimento de fundamental importância para a determinação da área de contato entre as partículas sólidas e a água, determinando preponderantemente a distribuição do diâmetro dos poros (Reichardt \& Timm, 2004).

Em elevados teores de água (baixas tensões) onde os fenômenos de capilaridade assumem grande importância na determinação do potencial mátrico, a curva de retenção de água fica na dependência da disposição e do tamanho dos poros, ou seja, a curva é função da estrutura do solo; em situações de baixos teores de água (altas tensões), o potencial mátrico praticamente independe dos fatores geométricos, sendo a estrutura de pouca importância em sua determinação (Reichardt, 1985).

A curva de retenção de água no solo é um indicador físico bastante valioso da qualidade do solo. Sua obtenção pode ser realizada de forma tradicional em laboratório utilizando amostras indeformadas ou deformadas de solo (Embrapa, 1997), fazendo uso de diferentes metodologias, dentre as quais o método da centrífuga (Silva \& Azevedo, 2002) e a clássica metodologia da mesa de tensão ou câmaras de pressão (Richards, 1965).

Tais métodos consistem no levantamento de certo número de pontos estrategicamente selecionados com os quais é traçada uma curva que relaciona a umidade volumétrica retida no solo em uma determinada tensão. Entretanto, tais métodos apresentam algumas limitações quanto à dificuldade no controle do ponto de equilíbrio entre a pressão aplicada e a água retida no solo. $\mathrm{O}$ uso de funções de pedotransferência (PTF) são alternativas interessantes para determinação da retenção da água no solo. Essas funções fazem uso da similaridade da retenção com a curva de distribuição de tamanho de partículas do solo. O método de Arya \& Paris (1981) foi testado e validado para vários solos brasileiros por Vaz et al. (2005). Neste trabalho são utilizadas análises granulométricas obtidas pelo equipamento baseado em atenuações de raios gama para estimar a retenção. Os métodos convencionais de análise granulométrica, pipeta e densímetro, não possibilitam a distribuição detalhada e contínua (curvas) dos diâmetros das partículas do solo; a porcentagem de areia é determinada à parte, por peneiramento (Crestana, 1994; Vaz et al., 1997). Outras limitações inerentes aos métodos convencionais são a elevada ocupação de espaço físico nos laboratórios, a forte dependência da habilidade da pessoa responsável por operar os equipamentos e o elevado prazo de resposta 
dos laboratórios que podem durar até meses para a determinação da curva de retenção (Vaz et al., 1999; Naime et al., 2001).

Dessa forma, o modelo de Arya \& Paris (1981) serviu de base para o desenvolvimento do software Qualisolo, que tem por objetivo adicionar novas funcionalidades ao analisador granulométrico automático (Vaz et al., 1999 e Naime et al., 2001), como a obtenção da curva de retenção de água no solo. O modelo desenvolvido por Arya \& Paris utiliza a similaridade entre as funções que descrevem a distribuição de tamanho de partículas e a curva de retenção da água no solo para obter essa última função indiretamente.

$\mathrm{O}$ analisador granulométrico tem como principal característica o fornecimento da distribuição detalhada do tamanho de partículas (DTP) que constitui o solo. Tal equipamento apresenta como principais vantagens a redução no tempo de determinação de 24 horas para 20 minutos, uma maior precisão na obtenção da curva completa da distribuição do tamanho de partículas e a não dependência da habilidade do operador, já que o processo de análise é automatizado (Vaz et al., 1999; Naime et al., 2001); com isso reduz-se as limitações existentes nos métodos tradicionais de determinação do tamanho das partículas minerais do solo, gerando uma nova alternativa de obtenção da curva de retenção, baseadas em parâmetros de fácil determinação e/ou disponíveis em levantamentos de solos (Naime et al., 2004).

Objetivou-se com esse trabalho a determinação e a comparação da curva de retenção de água no solo obtida pelos métodos proposto por Arya \& Paris (1981), método da câmara de Richards e método da centrífuga, em um Neossolo Quartzarênico do Perímetro Irrigado Senador Nilo Coelho, em Petrolina-PE.

\section{MATERIAL E MÉTODOS}

O estudo foi realizado em Petrolina-PE (latitude $09^{\circ} 30^{\prime} \mathrm{S}$; longitude $40^{\circ} 40^{\prime} \mathrm{W}$ e $365 \mathrm{~m}$ de altitude). Conforme a classificação de Köppen a região do Submédio São Francisco apresenta clima do tipo BSwh' (Reddy \& Amorim Neto, 1993). A fazenda Sasaki, localizada no lote 180 do Perímetro Irrigado Senador Nilo Coelho, Núcleo 5, apresenta um solo classificado como Neossolo Quartzarênico (Embrapa, 2006).

Para a realização do estudo foi selecionada uma área (área $K$, com 3,2 ha, e 40 fileiras de plantas e 41 plantas por fileiras) com um pomar de videira cultivar Crimson Seedless sobre o porta-enxerto IAC 313, plantada em setembro de 2005, no espaçamento de 5 x $4 \mathrm{~m}$ e irrigada por microaspersão, com 2 emissores por planta. Dentro da área $\mathrm{K}$, foi selecionada a subárea K2, com 0,8 ha, 10 fileiras de plantas e 41 plantas por fileiras, onde foram escolhidos três pontos do setor central da subárea para a coleta das amostras deformadas de solo com o auxílio de um trado, para a obtenção da curva de retenção de água no solo nas camadas de $0,00-0,20,0,20-0,40$ e $0,40-0,60 \mathrm{~m}$ de profundidade. Os solos coletados nos três pontos foram homogeneizados por camada, formando uma amostra composta para cada profundidade. Em seguida, tais amostras foram subdividas em três subamostras, as quais foram encaminhadas para a determinação da curva de retenção por diferentes metodologias. Nos mesmos três pontos, foram abertas pequenas trincheiras para a coleta, nas mesmas profundidades, de amostras indeformadas de solo, por meio de anéis volumétricos para a determinação da densidade global do solo $\left(\mathrm{d}_{\mathrm{g}}, \mathrm{kg} \mathrm{m}^{-3}\right)$, no Laboratório de Análise de Solo, Água e Planta da Embrapa Semiárido. Para o estabelecimento das curvas de retenção de água no solo, determinaram-se a tensão da água no solo (-MPa) e umidade do solo a base de massa (U, $\mathrm{kg}$ $\left.\mathrm{kg}^{-1}\right)$ das amostras, que foram transformadas em umidade do solo a base de volume $\left(\theta, \mathrm{m}^{3} \cdot \mathrm{m}^{-}\right.$ 
${ }^{3}$ ) pelo produto entre $\mathrm{U}$ e $\mathrm{d}_{\mathrm{g}}$. Estabeleceu-se a capacidade de campo a umidade correspondente a 0,006 $\mathrm{MPa}$ e ponto de murcha permanente a umidade a $1,5 \mathrm{MPa}$, concordando com (Reichardt, 1988).

Para todas as metodologias de determinação da curva de retenção empregadas nesse estudo foram utilizadas amostras deformadas de solo, devido às dificuldades de obtenção de amostras indeformadas dada à natureza do solo (arenoso) e de transporte das mesmas amostras do local do experimento para os laboratórios em Petrolina - PE, Botucatu - SP e São Carlos - SP, sem que ocorresse destruição do solo indeformado.

A análise granulométrica do solo da área experimental, realizada pelo método da pipeta (Embrapa, 1997) para as profundidades de 0-00,20, 0,20-0,40 e 0,40-0,60 m no Laboratório de Análise de Solo, Água e Planta da Embrapa Semiárido, está apresentada na Tabela 1.

Tabela 1. Analise granulométrica nas três profundidades de estudo.

\begin{tabular}{cccccc}
\hline $\begin{array}{c}\text { Profundidade } \\
(\mathbf{m})\end{array}$ & \multicolumn{5}{c}{ Granulometria $\left(\mathbf{g ~ K g}^{-\mathbf{1}}\right)$} \\
\cline { 2 - 6 } & AT & AG & AF & Silte & Argila \\
\hline $\mathbf{0 , 0 0 - 0 , 2 0}$ & 938,1 & 553,1 & 385,0 & 6,3 & 55,6 \\
$\mathbf{0 , 2 0 - 0 , 4 0}$ & 935,4 & 494,3 & 441,2 & 6,4 & 58,2 \\
$\mathbf{0 , 4 0 - 0 , 6 0}$ & 907,4 & 479,3 & 428,1 & 18,7 & 73,9 \\
\hline
\end{tabular}

$\mathrm{AT}=$ areia total; $\mathrm{AG}=$ areia grossa; $\mathrm{AF}=$ areia fina.

\subsection{Método da Câmara de Richards (R)}

No Laboratório de Física do Solo do Departamento de Recursos Naturais, Área de Ciência do Solo, da Faculdade de Ciências Agronômicas (FCA/UNESP), campus de Botucatu, a determinação da relação entre o potencial mátrico e a quantidade de água presente no solo pelo método da câmara de Richards foi realizada às tensões de 0,$006 ; 0,01 ; 0,033$; 0,$06 ; 0,1$ e 1,5 MPa.

\subsection{Método da Centrífuga (C)}

A obtenção da relação entre o potencial mátrico e a quantidade de água presente no solo por meio do método da centrífuga foi realizada no Laboratório de Análise de Solo, Água e Planta da Embrapa Semiárido, em Petrolina - PE, onde as amostras das camadas de 0,000,$20 ; 0,20-0,40$ e $0,40-0,60 \mathrm{~m}$ foram submetidas às rotações de $600,800,1.400,2.000,2.400$ e $9.200 \mathrm{rpm}$, correspondentes às tensões médias de 0,$006 ; 0,01 ; 0,03 ; 0,06 ; 0,1$ e $1,5 \mathrm{MPa}$. Para tanto foi utilizado um período de centrifugação de 3 horas (Silva \& Azevedo, 2002).

\subsection{Método de Arya \& Paris (AP)}

No método de Arya \& Paris (1981) a curva de retenção é obtida por meio do software Qualisolo (Naime et al., 2004), que utiliza como fundamento a distribuição do tamanho das partículas (DTP) proveniente do analisador granulométrico, desenvolvido e construído na Embrapa Instrumentação Agropecuária (Vaz et al., 1999; Naime et al., 2001).

A metodologia que subsidia a determinação da DTP do solo toma por base a atenuação de um feixe de raios gama - Lei de Beer-Lambert - (Eq. 1), utilizada para calcular a concentração (Eq. 1) em diferentes alturas da amostra (Naime et al., 2001). Assim: 


$$
\mathrm{c}=\frac{\ln \left(\frac{\mathrm{Io}}{\mathrm{I}}\right)}{\mathrm{x}\left(\mu_{\mathrm{p}}^{*}-\frac{\mu_{\mathrm{w}}^{*} \mathrm{D} \mathrm{w}}{\mathrm{D}}\right)}
$$

em que:

C - concentração em diferentes alturas da amostra;

$\mathrm{x}$ - espessura interna do recipiente;

Io - número de fótons que cruza o recipiente quando ele está preenchido com água (contagem por segundo, cps);

I - número de fótons que cruza o recipiente quando ele está com solução de solo (contagem por segundo, cps).

${ }^{*} \mu$ p e * $\mu$ w - são os coeficientes de atenuação em massa do solo e água $\left(\mathrm{m}^{2} \mathrm{~kg}^{-1}\right)$, respectivamente.

Dw e Dp - densidade de partículas do solo e água $\left(\mathrm{kg} \mathrm{m}^{-3}\right)$, respectivamente.

Para a granulometria esse método é acrescido do princípio da sedimentação de partículas em um meio líquido - lei de Stokes - (Eq. 2) o qual depende da viscosidade e da aceleração da gravidade (Vaz et al., 1997). Assim:

$$
t=\left(\frac{18 h \eta}{d^{2} g\left(D_{p}-D_{w}\right)}\right)
$$

em que:

$\mathrm{t}$ - tempo em que devem ser feita as medidas da atenuação para calcular o diâmetro $\mathrm{d}$ da partícula (s);

$\mathrm{h}$ - altura em que devem ser feita as medidas da atenuação para calcular o diâmetro d da partícula $(\mathrm{cm})$;

$\eta$ - viscosidade da água (poise);

d - diâmetro das partículas correlacionadas a concentração (m);

$\mathrm{g}$ - aceleração da gravidade $\left(\mathrm{cm} / \mathrm{s}^{2}\right)$;

Dw e Dp - densidade de partículas do solo e água $\left(\mathrm{g} / \mathrm{cm}^{3}\right)$, respectivamente.

No laboratório, as amostras de cada profundidade foram secas à temperatura ambiente, peneiradas em malha de $2 \mathrm{~mm}$ (Embrapa, 1997). Posteriormente, foi determinado o coeficiente de atenuação em massa (Vaz et al., 1997).

Após 24 horas em estufa a $105^{\circ} \mathrm{C}$, o solo foi pesado e seu valor inserido como um dos dados de entrada do software para execução das análises granulométricas. A determinação do coeficiente de atenuação em massa foi obtida preenchendo-se um recipiente retangular de acrílico $(0,05 \times 0,05 \times 0,08 \mathrm{~m})$ com solo previamente seco e peneirado de cada profundidade; 
esse recipiente foi pesado ainda vazio e medido o número de fótons transmitidos através do recipiente vazio (Io) e com solo (I) e a espessura $(\mathrm{x}, \mathrm{cm})$ que o feixe percorre no interior da amostra (Pedrotti et al., 2003). Para isso utilizou-se uma sub-rotina do programa para obtenção da distribuição do tamanho de partículas (Vaz et al., 1997).

As dispersões químicas das partículas de solo, de acordo com a metodologia descrita por Vaz et al. (1997), consistem, respectivamente, em adicionar $10 \mathrm{~mL}$ de $\mathrm{NaOH}(1 \mathrm{~N})$ em cada alíquota de solo e deixado em repouso por no mínimo 12 horas (Figura 2), procedendose à dispersão das partículas de solo em um agitador, modelo 936-2 da Hamilton Beach, em média rotação por 15 minutos.

Após esse processo, a solução contendo partículas de solo, água e $\mathrm{NaOH}$, foi transferida para recipientes retangulares de acrílico $(0,05 \times 0,05 \times 0,2 \mathrm{~m})$, acrescentando-se água destilada até que a solução atingisse a concentração de 100 g.L- ${ }^{1}$, ou seja, 0,16 m de altura (Vaz et al., 1999).

Os recipientes contendo essa solução foram colocados no analisador granulométrico para medidas de atenuação da radiação em intervalos de espaço e tempo previamente definidos, em diferentes alturas do recipiente contendo as partículas em sedimentação (Oliveira et al., 1997).

Na Figura (1) é apresentada a interface do programa Qualisolo; no canto superior esquerdo da tela principal são apresentados, em forma de tabela, os dados da análise granulométrica (concentração x diâmetro de partículas) e os 20 pares de pontos $(h, \theta)$ calculados da curva de retenção pelo método AP.

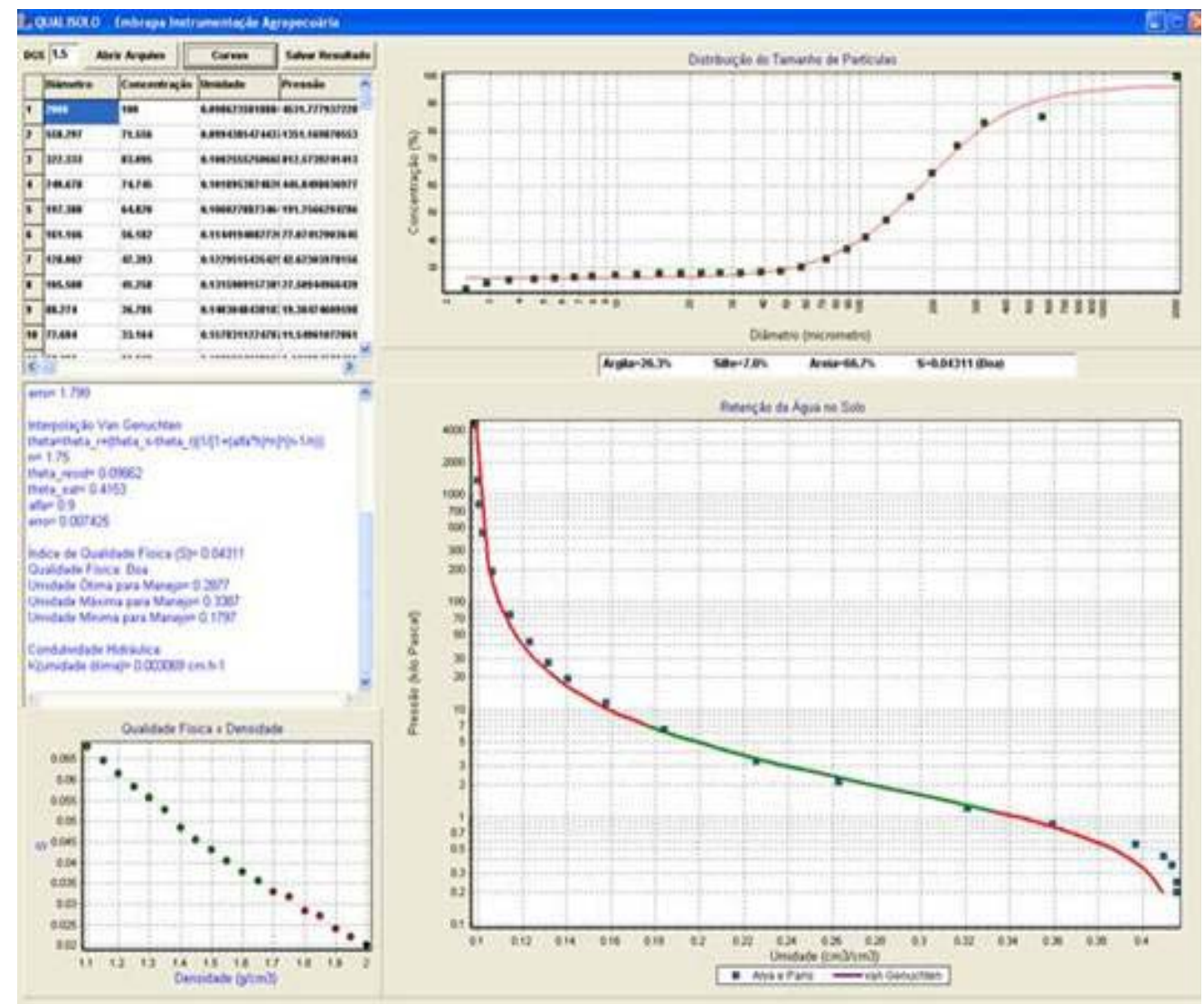

Figura 1. Detalhes da interface do programa Qualisolo (Naime et al., 2004). 
Na mesma figura, e no quadro abaixo, são apresentados os parâmetros da interpolação sigmoidal da distribuição de tamanhos de partículas (DTP), os parâmetros da equação do modelo de van Genuchten (1980), S, a condutividade hidráulica na umidade ótima de manejo e as umidades limite inferior, ótima e limite superior de manejo. A DTP é apresentada no gráfico superior indicando os pontos medidos pelo analisador e representando a função sigmoidal. Na curva de retenção são mostrados os 20 pontos calculados pelo método AP e a interpolação na equação do modelo de van Genuchten (1980). Durante os cálculos, o programa ajusta todos os parâmetros dos modelos de interpolação de forma a minimizar o erro médio quadrático em relação aos pontos experimentais, para o caso da análise granulométrica, ou em relação aos pontos do método AP para os parâmetros da equação de van Genuchten (Naime et al., 2006).

O modelo desenvolvido por van Genutchen (1980) foi utilizado para ajustar os dados obtidos a partir dos resultados observados pelos métodos da câmara de Richards e da centrífuga. Para tanto, se fez uso do software "Soil Water Retention Curve, versão Beta 3.0" SWRC (Dourado Neto et al., 2000). Para o método Arya \& Paris, tais parâmetros já são fornecidos e ajustados ao modelo de van Genuchten (Eq. 3) pelo aplicativo Qualisolo. Portanto:

$$
\theta=\theta_{\mathrm{r}}+\frac{\theta_{\mathrm{S}}-\theta_{\mathrm{r}}}{\left[1+\left|\alpha \Psi_{\mathrm{m}}\right|^{\mathrm{n}}\right]^{\mathrm{m}}}
$$

em que:

$\theta$ r . Umidade volumétrica residual $\left(\mathrm{m}^{3} \mathrm{~m}^{-3}\right)$;

$\theta$ s . Umidade volumétrica de saturação $\left(\mathrm{m}^{3} \mathrm{~m}^{-3}\right)$;

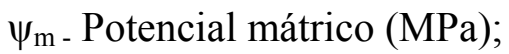

$\alpha$, m e n - Parâmetros empíricos da equação.

\section{RESULTADOS E DISCUSSÃO}

De acordo com a Figura 2, que fornece o ajuste das curvas características de água no solo pelos métodos de Arya \& Paris (AP), câmara de Richards (R) e da centrífuga (C) nas camadas de $0,00-0,20 ; 0,20-0,40$ e $0,40-0,60 \mathrm{~m}$ de profundidade foi possível observar uma boa similaridade entre os resultados obtidos pelos métodos $\mathrm{C}$ e $\mathrm{R}$ para as profundidades e tensões avaliadas, comportamento também observado por Centurion et al.,(1997).

$\mathrm{Na}$ comparação entre as três metodologias, verifica-se uma maior proximidade no comportamento das curvas para valores de tensão superiores a $0,1 \mathrm{MPa}$, ou seja, no intervalo de tensão onde a maior dependência no comportamento da curva está vinculada a textura e a superfície especifica das partículas, tal comportamento também verificado por Feliciano (2005) ao analisar métodos alternativos para obtenção da curva de retenção da água no solo. Dessa forma o efeito da estrutura sobre a retenção de água vai sendo reduzido, e tem-se um aumento acentuado do efeito da granulometria do solo (Urach, 2007). 


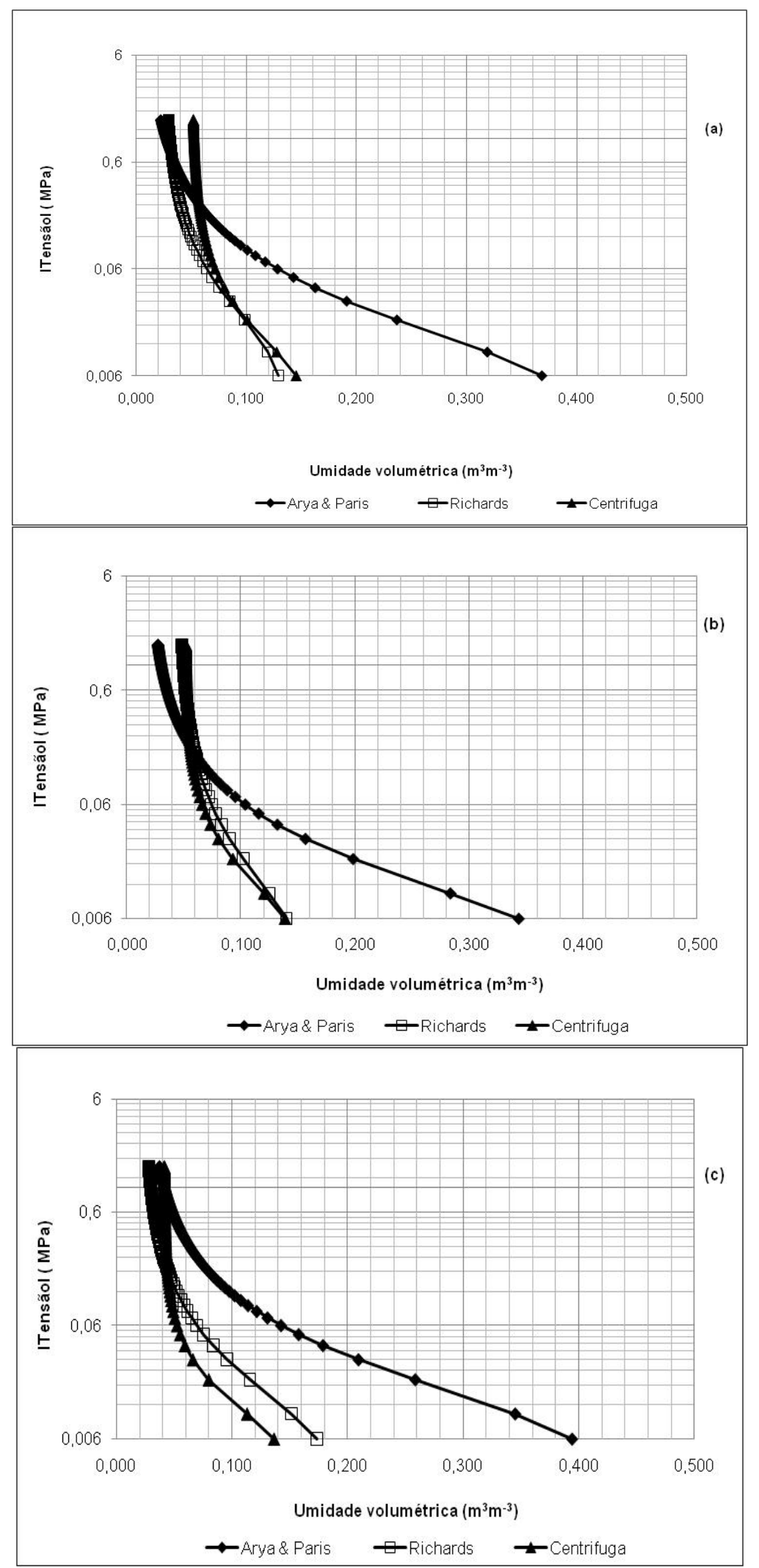

Figura 2. Curvas de retenção obtidas pelos métodos Arya \& Paris (AP), câmara de Richards (R) e centrífuga (C), nas camadas de 0,00-0,20 m (a); 0,20-0,40 m (b) e 0,40-0,60 m de profundidade (c). 
Os resultados da análise da curva proveniente da metodologia desenvolvida por Arya \& Paris (1981) permite verificar uma similaridade na quantidade de água retida em função da tensão aplicada para todas as profundidades em estudo, sendo tal comportamento mais uniforme para tensões superiores a $0,1 \mathrm{MPa}$. Essa situação é compreensível já que em elevados potenciais matriciais de água no solo, as forças capilares são mais atuantes e nessa faixa de potencial a textura e a estrutura do solo determinam a quantidade de água retida pelos poros do solo. Conforme Urach (2007), com a redução gradativa do potencial mátrico diminuem as forças capilares e aumentam os fenômenos de adsorção da água no solo pela diminuição da espessura do envelope que envolve as partículas do solo e aumenta a energia de retenção da água.

A granulometria altamente arenosa do solo em estudo é um fator que exerce grande influencia no comportamento da curva de retenção de água. Segundo Buckman \& Brady (1979), a areia possui reduzida capacidade de retenção de água causada pelo grande espaço entre as partículas granulométricas e o rápido escoamento de água de percolação.

Em baixas tensões ( 0 a $0,1 \mathrm{MPa})$ o comportamento da curva adquirida pelo método AP apresentou um comportamento discrepante ao verificado pelos métodos $\mathrm{R}$ e $\mathrm{C}$ para as mesmas tensões aplicadas.

De uma maneira geral para as três metodologias (Figura 2), pode-se verificar uma maior retenção de água para tensões inferiores a $0,01 \mathrm{MPa}$. Tais resultados corroboram com as observações de Urach (2007), ao avaliar estimativas da retenção para fins de irrigação, onde ele observou um efeito negativo da areia sobre a retenção de água, indicando que solos arenosos possuem menor capacidade de reter água.

A Figura 3 mostra a correlação entre umidades estimadas pelos métodos $\mathrm{R}$ x AP, C x AP e C x R para a camada de 0,00 - 0,20 m. Todas as comparações de métodos testados obtiveram alto coeficiente de correlação com $\mathrm{R}^{2}$ superiores á 0, 975. Segundo Urach (2007), em relações do tipo 1:1 a precisão do resultado é maior quanto menos pontos dispersarem em relação à linha principal, a qual representaria o ajuste perfeito. Da correlação R x AP (Figura 3a), é possível observar uma superestimação dos valores observados pelo método AP em relação aos determinados por $\mathrm{R}$, comportamento similar também é observado na correlação $\mathrm{C}$ $\mathrm{x}$ AP (Figura 3b), onde a variação na umidade em AP é superior a verificada pela C superestimando assim tal metodologia, a correlação C x R (Figura 3c), apresentou a melhor concordância entre os métodos testados, sendo, portanto mais precisa a correlação existente entre tais métodos, com uma leve superestimação do método $\mathrm{C}$ em relação ao método $\mathrm{R}$ em valores mais altos de umidade.

Nas camadas de 0,20-0,40 m e 0,40 a 0,60 m de profundidade (Figuras 4 e 5) é possível observar uma tendência similar no comportamento da correlação da umidade entre os métodos testados, sendo constante a superestimação da umidade obtida pelo método AP em comparação aos métodos da C e de R. A correlação entre os métodos R x C (Figura 4 e 5 c) apresenta uma maior precisão dos dados calculados, caracterizando-se também por apresentar uma pequena variação no intervalo de dados de umidade, quando se compara com as correlações provenientes dos métodos AP x R (Figura 4 e 5 a) e AP x C (Figura 4 e 5 b), no entanto observou-se uma leve superestimação do método de $\mathrm{R}$ em relação a metodologia da C. 


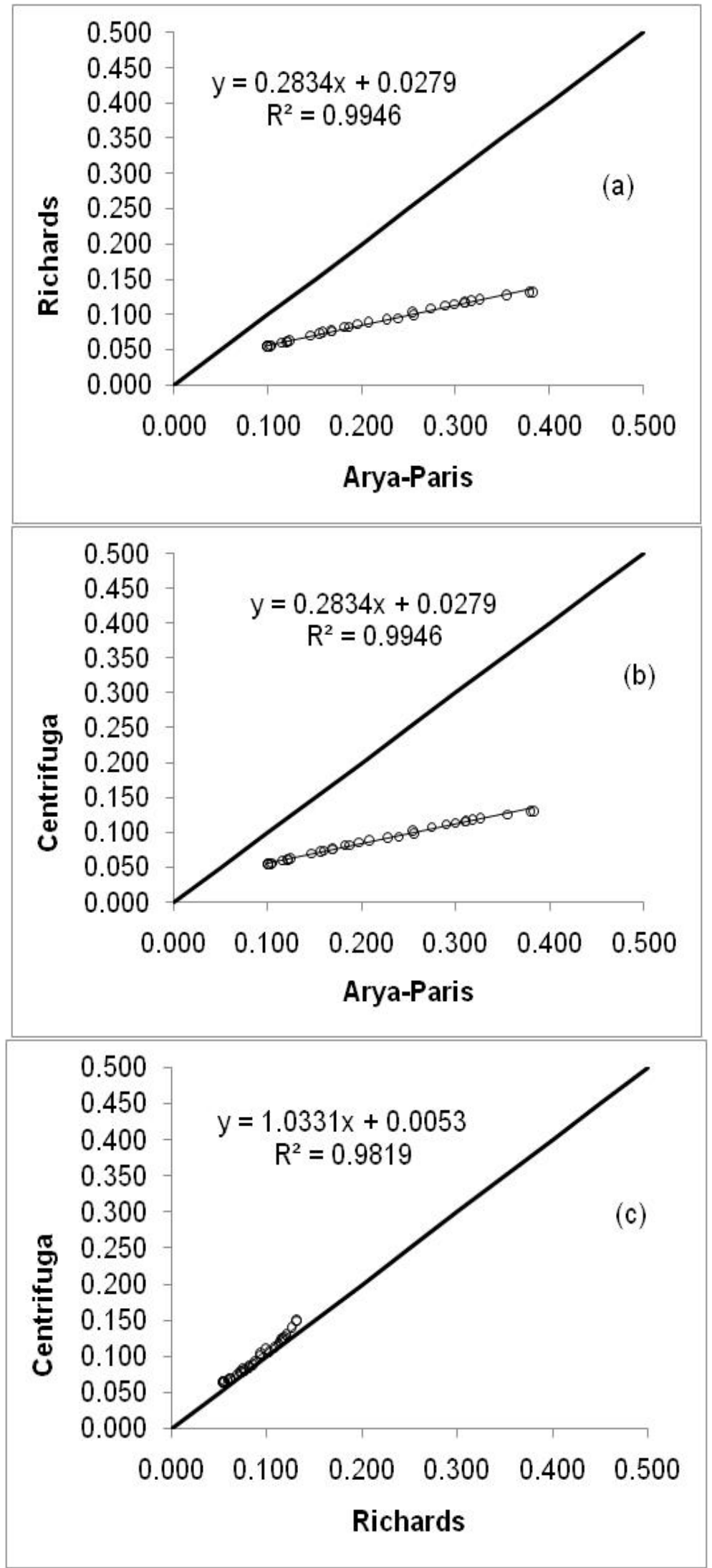

Figura 3. Correlação entre umidades calculada para os métodos da câmara de Richards $x$ Arya \& Paris (a), centrífuga x Arya \& Paris (b) e centrífuga x câmara de Richards (c), para a camada de $0,00-0,20 \mathrm{~m}$ de profundidade. 


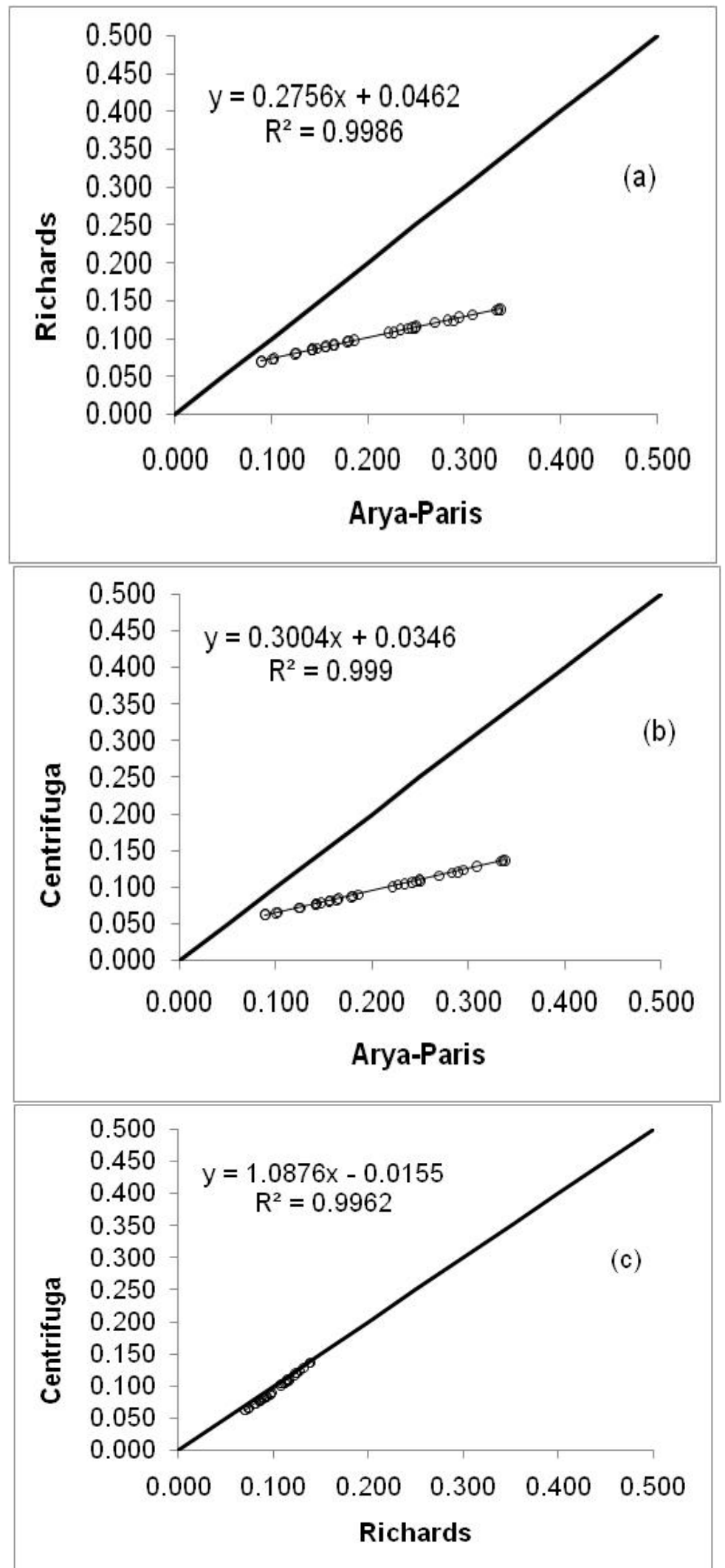

Figura 4. Correlação entre umidades calculada para os métodos da câmara de Richards $x$ Arya \& Paris (a), centrífuga x Arya \& Paris (b) e centrífuga x câmara de Richards (c), para a camada de 0,20-0,40 $\mathrm{m}$ de profundidade. 


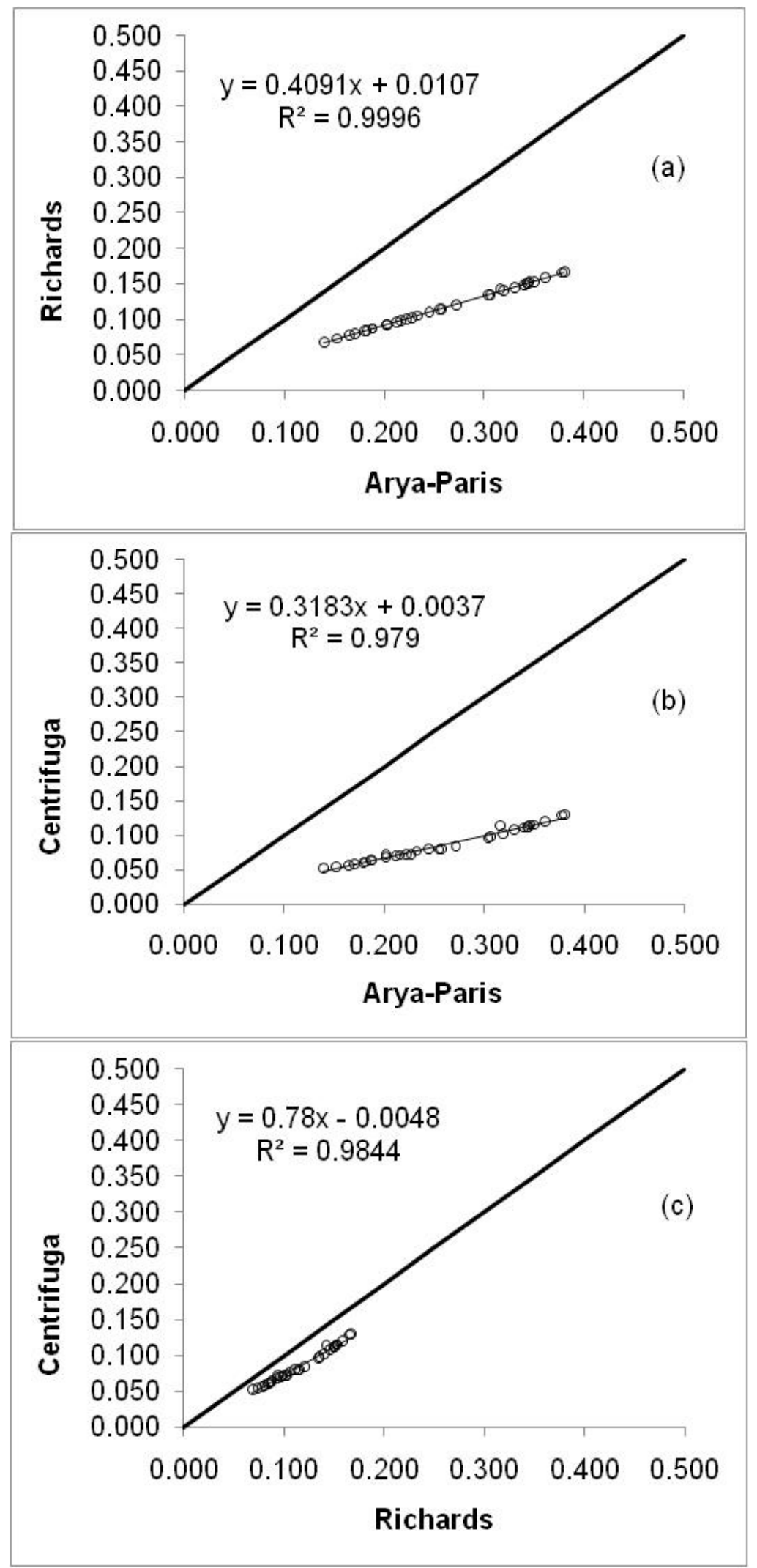

Figura 5. Correlação entre umidades calculada para os métodos da câmara de Richards $x$ Arya \& Paris (a), centrífuga x Arya \& Paris (b) e centrífuga x câmara de Richards (c), para a camada de 0,40-0,60 $\mathrm{m}$ de profundidade. 
A utilização do método AP para descrever retenção e a umidade do solo não conseguiu obter correspondência com nenhum dos métodos tradicionalmente utilizados $(\mathrm{C}$ e $\mathrm{R})$. Tal desempenho pode estar atribuído a descrição minuciosa da granulometria utilizada para a obtenção da curva de retenção pelo método AP, já que o mesmo faz uso do analisador granulométrico de partículas do solo que tem por principio o fornecimento de resultados padronizados e com boa precisão. Outro fato a se destacar é que no sistema de produção de uva no Submédio São Francisco, a aplicação de composto e/ou esterco para aumentar a quantidade de matéria orgânica do solo é prática comum. $\mathrm{Na}$ área em questão, a aplicação é feita anualmente, por meio de aberturas de covas até $0,6 \mathrm{~m}$ de profundidade, ao lado do tronco da videira. O local da abertura de cova varia de um ano para outro, a fim de permitir uma melhoria do solo ao redor de toda a planta. Essa aplicação de composto e/ou esterco pode ter contribuído para uma maior quantidade de partículas de menor tamanho (matéria orgânica) no solo em estudo.

Para Skopp (2000), a distribuição do tamanho de partículas, é a propriedade física mais importante de um solo, influenciando na maioria de suas propriedades físicas. A divergência de dados verificada ao relacionar os métodos da $\mathrm{C}$ e $\mathrm{R}$ com a metodologia desenvolvida por AP também pode estar atribuída às diferenças granulométricas entre o solo utilizado por Vaz et al. (2005), durante a elaboração da função de pedotransferência que descreve a curva de retenção pelo método AP e a granulometria do solo onde foi desenvolvido o presente estudo. Essa situação também verificada por Urach (2007), ao concluir que as funções de pedotransferência são mais eficientes quando utilizadas para estimar a retenção em solos com características similares aos utilizados na geração das equações.

\section{CONCLUSÕES}

Os valores de umidade do solo estimados pela curva de retenção de água de um Neossolo Quartzarênico obtida pelo método proposto por Arya \& Paris foram superiores aos valores de umidade determinados pelos métodos da câmara de Richards e da centrífuga, à mesma tensão. Esses dois últimos métodos apresentaram boa similaridade. No entanto, recomenda-se o desenvolvimento de calibrações específicas do método de Arya \& Paris para os solos irrigados do Submédio São Francisco, devido a possibilidade de uso da curva de retenção de água no solo para o manejo de irrigação e pelo potencial do método quanto à determinação rápida e exata.

\section{REFERÊNCIAS BIBLIOGRÁFICAS}

ARYA, L. M.; PARIS, J. F. A physicoempirical model to predict soil moisture characteristics from particle-size distribution and bulk density data. Soil Science Society of America Journal, Madison, v. 45, p. 1023-1030, 1981.

BUCKMAN, H. O.; BRADY, N. C. Natureza e propriedade dos solos. 5. ed. Rio de Janeiro: Biblioteca Universitária Freitas Bastos, 1979. 647 p.

CENTURION, J. F.; MORAES, M. H.; DELLA LIBERA, C. L. F. Comparação de métodos para determinação da curva de retenção de água em solos. Revista Brasileira de Ciência do Solo, Campinas, v. 21, p. 173-179, 1997. 
CRESTANA, S. Técnicas recentes de determinação de características do solo. In: REUNIÃO BRASILEIRA DE MANEJO E CONSERVAÇÃO DO SOLO E DA ÁGUA, 10., 1994, Florianópolis, SC.. Resumos... Florianópolis: SBCS, 1994. p. 86-97.

DOURADO-NETO, D. et al. Programa computacional para modelagem de curvas de retenção de água no solo (SWRC, versão 2.00). Sciência Agricola, Piracicaba, v. 57, n. 1, Mar. 2000.

EMBRAPA. Centro Nacional de Pesquisa de Solos. Sistema Brasileiro de Classificação de Solos. Rio de Janeiro, RJ,: Embrapa Solos, 2006. 2a ed. 306p.

EMBRAPA. Manual de métodos de análise de solo. Rio de Janeiro: Centro Nacional de Pesquisa de Solos, 1997. 212 p.

FELICIANO, J. J. S. Métodos alternativos para obtenção da curva de retenção da água no solo. 2005. 103 p. Dissertação (Mestrado em Engenharia Agrícola) - Universidade Estadual do Oeste do Paraná, Cascavel, 2005.

MARCIANO, C. R. et al. Variabilidade do potencial mátrico e do conteúdo de água no solo em experimento de manejo de irrigação. Revista Brasileira de Ciência do Solo, Viçosa, MG, v. 22 , n. 4, p. 563-571, 1998.

NAIME, J. M.; VAZ, C. M. P.; MACEDO, A. Automated soil particle size analyzer based on gamma-ray attenuation. Computers and Electronics in Agriculture, Amsterdam, v. 31, n. 3, p. 295-304, 2001.

NAIME, J. de M.; VAZ, C. M. P.; MACEDO, Á. Determinações físicas do solo a partir da granulometria: Programa Qualisolo. In: REUNIÃO BRASILEIRA DE MANEJO E CONSERVAÇÃO DO SOLO E DA ÁGUA, 16., Aracaju, 2006. Resumos e palestras... Aracaju: Sociedade Brasileira de Ciência do Solo, 2006. 1 CD-ROM.

NAIME, J. M.; SHINYA, V. T.; VAZ, C. M. P. Programa para estimativa indireta da curva de retenção da água no solo. In: REUNIÃO BRASILEIRA DE MANEJO E CONSERVAÇÃO DO SOLO E DA ÁGUA, 15., 2004, Santa Maria, RS. Anais... Santa Maria: UFSM, 2004. 1 CD-ROM.

OLIVEIRA, J. C. M. et al. Improved soil particle-size analysis by gamma-ray attenuation. Soil Science Society of America Journal, Madison, v. 61, p. 23-26, 1997.

PEDROTTI, A. et al. Relação entre atributos físicos, mineralogia da fração argila e formas de alumínio no solo. Revista Brasileira de Ciência do Solo, Viçosa, MG, v. 27, p. 1-9, 2003.

REDDY, S. J.; AMORIM NETO, M. S. Dados de precipitação, evapotranspiração potencial, radiação solar global de alguns locais e classificação climática do Nordeste do Brasil. Petrolina, PE: EMBRAPA, CPATSA, 1993. 280 p.

REICHARDT, K. Capacidade de campo. Revista Brasileira de Ciência do Solo, Campinas, v. 12, p. 211-216, 1988. 
REICHARDT, K. Processos de transferência no sistema solo-planta-atmosfera. Campinas: Fundação Cargill, 1985. 466 p.

REICHARDT, K.; TIMM, L. C. Solo, planta e atmosfera: conceitos, processos e aplicações. Piracicaba: Manole, 2004. 478 p.

RICHARDS, L. A. Physical conditions of water in soil. In: BLACK, C. A. et al. (Eds.). Methods of soil analysis: physical and mineralogical properties, including statistics of measurements and sampling. Madison: American Society of Agronomy, 1965. p. 128-152.

SKOPP, J. M. Physical properties of primary particles. In: SUMNER, M. (Ed.). Handbook of soil science. Boca Ratón: CRC, 2000. p. 3-17.

SILVA, E. M. da; AZEVEDO, J. A. de. Influência do período de centrifugação na curva de retenção de água em solos de Cerrado. Pesquisa Agropecuária Brasileira, Brasília, v. 37, p. 1487-1494, 2002.

URACH, F. L. Estimativa da retenção de água em solos para fins de irrigação. 2007. 79 p. Dissertação (Mestrado em Engenharia Agrícola)-Universidade Federal de Santa Maria, Santa Maria, 2007.

VAN GENUCHTEN, M. T. A closed form equation for predicting the hydraulic conductivity of unsaturated soils. Soil Science Society of America Journal, Madison, v. 44, p. 892-898, 1980.

VAZ, C. M. P.; NAIME, J. M.; MACEDO, A. Soil particle size fractions determined by gamma-ray attenuation. Soil Science, Baltimore, v. 164, n. 6, p. 403-410, 1999.

VAZ, C. M. P. et al. Análise granulométrica por raios gama. São Carlos: EMBRAPA, CNPDIA, 1997. 13 p. (Boletim de pesquisa, n.5).

VAZ, C. M. P. et al. Validation of the Arya and Paris water retention model for Brazilian soils. Soil Science Society of America Journal, Madison, v. 69, p. 577-583, 2005.

VIEIRA, M. L. Propriedades físico-hídrico-mecânicas do solo e rendimento de milho submetido a diferentes sistemas de manejo. 2006. 115 p Dissertação (Mestrado em Agronomia) - Universidade de Passo Fundo, Passo Fundo, 2006. 